\title{
Atomic resolution 3D electron diffraction microscopy
}

Jianwei Miao*, Tetsu Ohsuna ${ }^{\dagger}$, Osamu Terasaki $i^{\ddagger} \&$ Michael A. O’Keefe ${ }^{\S}$

"Stanford Synchrotron Radiation Laboratory, Stanford Linear Accelerator Center, Stanford

University, Stanford, CA 94309-0210, USA

†Institute for Materials Research, Tohoku University, Sendai 980-8577, Japan

${ }^{*}$ Department of Physics and CIR, Tohoku University, Sendai 980-8578, Japan

${ }^{\S}$ Materials Science Division, National Center for Electron Microscopy, Lawrence Berkeley National Laboratory, Berkeley, CA 94720, USA

Electron lens aberration is the major barrier limiting the resolution of electron microscopy. The traditional approach to overcome the barrier is to use the holographic method originally proposed by Gabor in $1948^{1}$. Holography is a two-step imaging approach ${ }^{2}$, including (i) obtaining a hologram from known reference waves and unknown object waves, and (ii) converting the hologram to an image by using either light-optical or digital reconstruction. Here we describe a novel form of electron microscopy to overcome electron lens aberration. By combining the coherent electron diffraction with the oversampling phasing method, we show that the 3D structure of a $2 \times 2 \times 2$ unit cell nano-crystal (framework of LTA $\left[\mathrm{Al}_{12} \mathrm{Si}_{12} \mathrm{O}_{48}\right]_{8}$ ) can be $a b$ initio determined at the resolution of $1 \AA$ from a series of simulated noisy diffraction pattern projections with rotation angles ranging from $-70^{\circ}$ to $+70^{\circ}$ in $5^{\circ}$ increments along a single rotation axis. This form of microscopy (which we call 3D electron diffraction microscopy) does not require any reference waves, and can image the 3D structure of nano-crystals, as well as non-crystalline biological and materials science samples, with the resolution limited only by the quality of sample diffraction. 
When a beam of coherent electrons illuminates a finite sample, the electrons scattered by the Coulomb potential of the sample form a diffraction pattern in the far field. If the sample is thin enough so that the multiple scattering effect is negligible, the diffraction pattern is proportional to the square of the magnitude of the Fourier transform of the Coulomb potential. Due to the finite size of the sample, the diffraction pattern can be sampled at a spacing finer than the Nyquist frequency (i.e. the inverse of the size of the sample), which corresponds to surrounding the Coulomb potential of the sample with a region of zeros ${ }^{3,4}$. The finer the sampling frequency, the larger the region of zeros. To characterize the oversampling degree, a parameter of the oversampling ratio $(\sigma)$ has been introduced $^{4}$

$$
\sigma=\frac{\text { volume of Coulomb potential region }+ \text { volume of zeros region }}{\text { volume of Coulomb potential region }}
$$

where the Coulomb potential region and the region of zeros are separated by a finite support. When $\sigma>2$, the phases are in principle embedded inside the diffraction pattern, and can be directly retrieved by using an iterative algorithm ${ }^{5,6}$. The first experimental demonstration of the oversampling method was carried out in 1999 by using soft X-rays ${ }^{7}$. Recently, it has been applied to determine the 2D shapes of Au nano-crystals ${ }^{8}$ and the 3D structure of a non-crystalline sample ${ }^{9}$ by using coherent hard X-rays. Potential applications of the oversampling method to other fields have also been pursued elsewhere ${ }^{10}$. In this letter, we show that the combination of the coherent electron diffraction and the oversampling method can determine the $3 \mathrm{D}$ structures of nano-crystals and non-crystalline samples at atomic resolution.

Fig. 1 shows the schematic layout of a 3D electron diffraction microscope. A beam of coherent electrons is generated by an electron gun and is focused to a small spot by a 
lens. An aperture is placed in front of the lens to adjust the spatial coherence. The 3D electron diffraction microscopy requires coherence length longer or equal to the overall size of the Coulomb potential region and the region of zeros, which are correlated to the oversampling degree by

$$
\alpha \leq \frac{\lambda}{4 O a} \quad \text { and } \quad \frac{E}{\Delta E} \geq \frac{O a}{d}
$$

where $\alpha$ the semi-angle of illumination produced by the illumination aperture shown in Fig. $1, \lambda$ the wavelength of the electron beam, $E / \Delta E$ the relative energy spread of the electron beam, $d$ the desired resolution, and $O$ the oversampling degree which is equal to $\sqrt{\sigma}$ for a 2D sample with size of $a x a$ and $\sqrt[3]{\sigma}$ for a 3D sample with size of $a x a x a$. The sample is placed at the focal spot whereas an adjustable illumination area determines the size of the sample. The sample can be rotated along a single axis for the $3 \mathrm{D}$ structure determination. A 2D area detector (such as a CCD or an imaging plate) is placed downstream of the sample for obtaining the oversampled diffraction patterns. Each component is in vacuum with a pressure comparable to that of transmission electron microscopy.

In order to test this form of microscopy, a computer simulation experiment was carried out using a model nano-crystal (framework of LTA $\left[\mathrm{Al}_{12} \mathrm{Si}_{12} \mathrm{O}_{48}\right]_{8}$ ) with 2 × 2 × 2 unit cells. This structure has the cubic crystal system, with the space group Fm3c and the whole particle size of $49.22 \times 49.22 \times 49.22 \AA^{3}$ (ref. 11). The reason that this structure was chosen for the simulation is due to its complexity. Emphasis should be made here that the oversampling phasing method can not make use of the high symmetry of the structure since both the Bragg peaks and the intensity between the Bragg peaks are needed for the phase retrieval. Fig. 2a shows a slice ( $0.5 \AA$ thick) of the nano-crystal viewed along $[100]$ at $\mathrm{z}=0$, in which the Coulomb potential of $\mathrm{Si}, \mathrm{Al}$ and $\mathrm{O}$ atom was calculated by using a five- 
Gaussian approximation $^{12}$, and the Debye-Waller factor of $\mathrm{Si}, \mathrm{Al}$ and $\mathrm{O}$ atom was set at 0.3 , 0.3 and $0.5 \AA^{2}$, respectively. In the simulation, a beam of coherent electrons with the energy of $300 \mathrm{keV}$ was used and the oversampling ratio $(\sigma)$ was set at 4.3 in three dimensions. According to Eq. (2), the required spatial coherence of $\alpha \leq 6 \times 10^{-5} \mathrm{rad}$ and the required temporal coherence of $\mathrm{E} / \Delta \mathrm{E} \geq 80$ were estimated for the desired resolution of $1 \AA$, in which the temporal coherence can be easily satisfied since electron guns usually have $\mathrm{E} / \Delta \mathrm{E} \sim 10^{5}$. An electron beam with both the spatial and temporal coherence better than the required ones was simulated for the studies.

Since the multiple scattering from the thin sample is negligible, the diffraction pattern is proportional to the square of the magnitude of the Fourier transform of the Coulomb potential. By setting the rotation angles ranging from $-70^{\circ}$ to $+70^{\circ}$ in $5^{\circ}$ increments along the single rotation axis, a series of twenty-nine $2 \mathrm{D}$ diffraction patterns was calculated. To make the computer simulation practical, noise was added on each diffraction pattern with the signal-to-noise ratio of 3 . To simulate a beam stop, an area of 11 x 11 pixels was removed at the center for each diffraction pattern, but the value of the center pixel in the diffraction pattern with the rotation angle of $0^{\circ}$ remained unchanged. We have found that the reconstructions are very sensitive to the value of the center pixel of the diffraction pattern, which is likely because the value of the center pixel represents the sum of the Coulomb potential distribution. Fig. $2 b$ shows a diffraction pattern with a rotation angle of $0^{\circ}$ and a resolution of $1 \AA$ at the edge. The series of diffraction patterns were used to assemble a 3D magnitude of the Fourier transform with the array size of $160 \times 160 \times 160$ voxels where a voxel is the smallest element of a 3D array. Due to the limited number of diffraction pattern projections, the assembled 3D magnitude included a number of 
undefined voxels whose value could not be calculated from the diffraction pattern projections. Of a possible 4,096,000 voxels (160x160x160), our 29 diffraction pattern projections provided data for 702,880 voxels $(17.2 \%)$, leaving $3,393,120$ voxels $(82.8 \%)$ undefined.

This 3D magnitude can be directly converted to an image by using a 3D reconstruction algorithm without the need of interpolation. The algorithm first generated a $3 \mathrm{D}$ array of random phases. By combining the random phases with the $3 \mathrm{D}$ array of the known magnitudes, a new set of Fourier transform values was calculated. By applying the inverse fast Fourier transform to the 3D Fourier transform set, a 3D array of Coulomb potential was obtained. Based on the oversampling ratio, a box of 54 x 54 x $54 \AA^{3}$ was defined as the finite support, which is somewhat larger than the true envelope of the sample. Outside the finite support, the Coulomb potential was gradually pushed to zero. Inside the finite support, the negative Coulomb potential was pushed to zero and the positive Coulomb potential remained unchanged (see Methods). In this way, a new 3D array of Coulomb potential was obtained. By applying the fast Fourier transform to the new 3D array, a new 3D Fourier transform was calculated. The magnitude of the new 3D Fourier transform was then replaced with the $3 \mathrm{D}$ array of the known magnitude, in which the value of those undefined voxels in the new 3D Fourier transform remained unchanged. This represents one iteration of the algorithm. After about 2000 iterations, the correct phases were retrieved, which was indicated by an error function used for monitoring the reconstruction $^{5}$. The computation time of 2000 iteration on a $440 \mathrm{MHz}$ Sun Solaris computing workstation is about 8 hours. Fig. $2 \mathrm{c}$ shows a slice $(0.5 \AA$ thick $)$ of the reconstructed $3 \mathrm{D}$ Coulomb potential distribution viewed along [100] at $\mathrm{z}=0$, which is consistent to Fig. 2a. Fig. 2d shows an iso-surface rendering of one-eighth of the 
reconstructed Coulomb potential (one unit cell), in which the pores and cages of the nanocrystal can be seen. Five more reconstructions were carried out by using different random phase sets as the initial input. The reconstructed Coulomb potential distribution was consistent among all six, which demonstrated the robustness of this approach. Note that electron diffraction microscopy does not require the sample to be periodic. Although 3D electron crystallography has been shown to reveal the averaged 3D structure of periodic objects $^{13}$, electron diffraction microscopy reveals the local non-averaged 3D structure down to the single-atom level.

By using careful computer simulation, a form of microscopy, based on coherent electron diffraction and the oversampling phasing method, has been justified to overcome electron lens aberration. By employing this form of microscopy, the 3D structure of a $2 \times 2$ x 2 unit cell nano-crystal (framework of LTA $\left[\mathrm{Al}_{12} \mathrm{Si}_{12} \mathrm{O}_{48}\right]_{8}$ ) was ab initio determined at 1 $\AA$ resolution from a series of $2 \mathrm{D}$ diffraction pattern projections with the rotation angles ranging from $-70^{\circ}$ to $70^{\circ}$ in $5^{\circ}$ increments along a single rotation axis. This form of microscopy can be applied to determine the 3D structure of nano-crystals and noncrystalline materials science samples with the resolution limited only by the quality of sample diffraction. Unlike transmission electron microscopy (TEM), the 3D electron diffraction microscopy can easily bypass the diffraction/thickness limit by assembling a 3D diffraction pattern from a series of 2D diffraction patterns lying on the curved Ewald sphere. The 3D diffraction pattern can then be converted to a 3D image at sub-atomic resolution beyond the capability of TEM.

In biology, this form of microscopy can be used to image the 3D structure of cellular organelles, molecular clusters and single molecules with the ultimate resolution limited by radiation damage to the samples ${ }^{14}$. Compared with 3D electron tomography ${ }^{15,16}$, 
this form of microscopy does not require data interpolation (which reduces the potential artifacts in the images) or heavy atom markers to align the tilted projections, and imposes relatively lower radiation dosage to the samples for achieving the same resolution due to the severe fall-off of the high frequency information for lens-based images ${ }^{17}$.

\section{Methods}

\section{Imaging mechanism}

The imaging mechanism of 3D electron diffraction microscopy is somewhat different from that of transmission electron microscopy. The former reconstructs the small phase shift inside each resolution volume of the sample where a resolution volume is a sphere with the diameter corresponding to the resolution length, while the latter images the exit waves from the sample. Due to the small size of each resolution volume, a phase object for 3D electron diffraction microscopy is usually a weak phase object, which is not necessarily the case for transmission electron microscopy. In the computer simulation, we assumed that the absorption by the sample is negligible, which makes the small phase shift inside each resolution volume not only linear proportional to the Coulomb potential but also real and positive. When the absorption is not negligible, the phase shift becomes complex, but the real and imaginary part of the phase shift inside each resolution volume remain positive. While the computer simulation in this letter employed a sample with real and positive Coulomb potential, the oversampling phasing method works for the samples with complex Coulomb potential as well (see ref. 4 for the computer simulation of the successful reconstruction of complex electron density in X-ray diffraction by using the oversampling method). 
1. Gabor, D. A new microscopic principle. Nature 161, 777-778 (1948).

2. Tonomura, A. Electron Holography, Springer Ser. Opt. Sci., vol. 70 (Springer, Berlin, Heidelberg 1993).

3. Sayre, D. in Imaging Processes and Coherence in Physics. Springer Lecture Notes in Physics, vol. 112, M. Schlenker et al., Eds. (Berlin: Springer, 1980) pp. 229-235.

4. Miao, J., Sayre, D. \& Chapman, H. N. Phase retrieval from the magnitude of the Fourier transforms of non-periodic objects. J. Opt. Soc. Am. A 15, 1662-1669 (1998).

5. Miao, J., Kirz, J. \& Sayre, D. The oversampling phasing method. Acta Crystallogr. D 56, 1312-1315 (2000).

6. Fienup, J. R. Phase retrieval algorithm: a comparison. Appl. Opt. 21, 2758-2769 (1982).

7. Miao, J., Charalambous, P., Kirz, J. \& Sayre, D. Extending the methodology of Xray crystallography to allow imaging of micrometre-sized non-crystalline specimens. Nature 400, 342-344 (1999).

8. Robinson, I. K., Vartanyants, I. A., Williams, G. J., Pfeifer, J. A. \& Pitney, J. A. Reconstruction of the Shapes of Gold Nanocrystals using Coherent X-ray Diffraction. Phys. Rev. Lett. 87, 195505-195509(2001).

9. Miao, J. et al. High Resolution 3D X-ray Diffraction Microscopy. Submitted to Science.

10. Spence, J. C. H., Howells, M., Marks, L. D. \& Miao, J. Lensless imaging: a workshop on "new approaches to the phase problem for non-periodic objects". Ultramicroscopy 90, 1-6 (2001). 
11. Gramlich, V. \& Meier, W. M. The crystal structure of hydrated NaA: A detailed refinement of a pseudosymmetric zeolite structure. Z. Kristallogr. 133, 134-149 (1971).

12. Peng, L. M., Ren, G., Dudarev, S. L., \& Whelan, M. J. Robust Parameterization of Elastic and Absorptive Electron Atomic Scattering Factors. Acta Crystallogr. A 52, 257-276 (1996).

13. Downing, K. H., Hu, M., Wenk, H. \& O'Keefe, M. A. Resolution of oxygen atoms in staurolite by three-dimensional transmission electron microscopy. Nature $\mathbf{3 4 8}$, $525-528(1990)$.

14. Henderson, R. Q. The potential and limitations of neutrons, electrons, and X-rays for atomic resolution microscopy of unstained biological molecules. Q. Rev. Biophys. 28, 171-193 (1995).

15. Crowther, R. A., DeRosier, D. J. \& Klug, A. The reconstruction of a threedimensional structure from projections and its application to electron microscopy. Proc. Roy. Soc. Lond. A. 317, 319-340 (1970).

16. Frank, J. Electron Tomography, Plenum, New York (1992).

17. Baldwin, J. M., Henderson, R., Beckman, E. \& Zemlin, F. Images of Purple Membrane at $2.8 \AA$ Resolution Obtained by Cryo-electron Microscopy. J. Mol. Biol. 202, 585-591 (1998).

Acknowledgements. J. M. thanks J. C. H. Spence for many stimulating discussions, and K. O. Hodgson for his encouragement and support. This work was supported by the Stanford Synchrotron Radiation Laboratory, which is operated by the U.S. Department of Energy, Office of Basic Energy Sciences, by CREST, JST (Japan Science and Technology Corporation), and by the Director, Office of Science, through the Office of Basic Energy Sciences, Material Sciences Division, of the U.S. Department of Energy.

\section{Correspondence and requests for materials should be addressed to J. M. (e-mail:}

\section{miao@slac.stanford.edu).}


FIG. 1. Schematic layout of a 3D electron diffraction microscope.

FIG. 2. 3D structure determination of a nano-crystal (framework of LTA $\left[\mathrm{Al}_{12} \mathrm{Si}_{12} \mathrm{O}_{48}\right]_{8}$ ) with $2 \times 2 \times 2$ unit cells from a series of diffraction pattern projections with the rotation angles ranging from $-75^{\circ}$ to $75^{\circ}$ in $5^{\circ}$ increments along a single rotation axis. a, A slice ( $0.5 \AA$ thick) of the $2 \times 2 \times 2$ unit cell nano-crystal viewed along [100] at $z=0$ with the image size of $49.22 \times 49.22 \AA^{2}$. b, The diffraction pattern with the rotation angle of $0^{\circ}$, in which the center square represents the missing data due to a simulated beam stop (except for the value of the center pixel). Both the Bragg peaks and the intensity between the Bragg peaks were "recorded". The resolution at the edge corresponds to $1 \AA$. c, The reconstructed slice (0.5 $\AA$ thick) of the $2 \times 2 \times 2$ unit cell nano-crystal viewed along [100] at $z=0$ with the image size of $49.22 \times 49.22 \AA^{2}$. d, An iso-surface rendering of one-eighth of the reconstructed 3D Coulomb potential distribution (one unit cell) with the image size of $24.61 \times 24.61 \times 24.61 \AA^{3}$, where the large spheres represent either the $\mathrm{Al}$ or $\mathrm{Si}$ atoms, and the smaller ones the $\mathrm{O}$ atoms. 


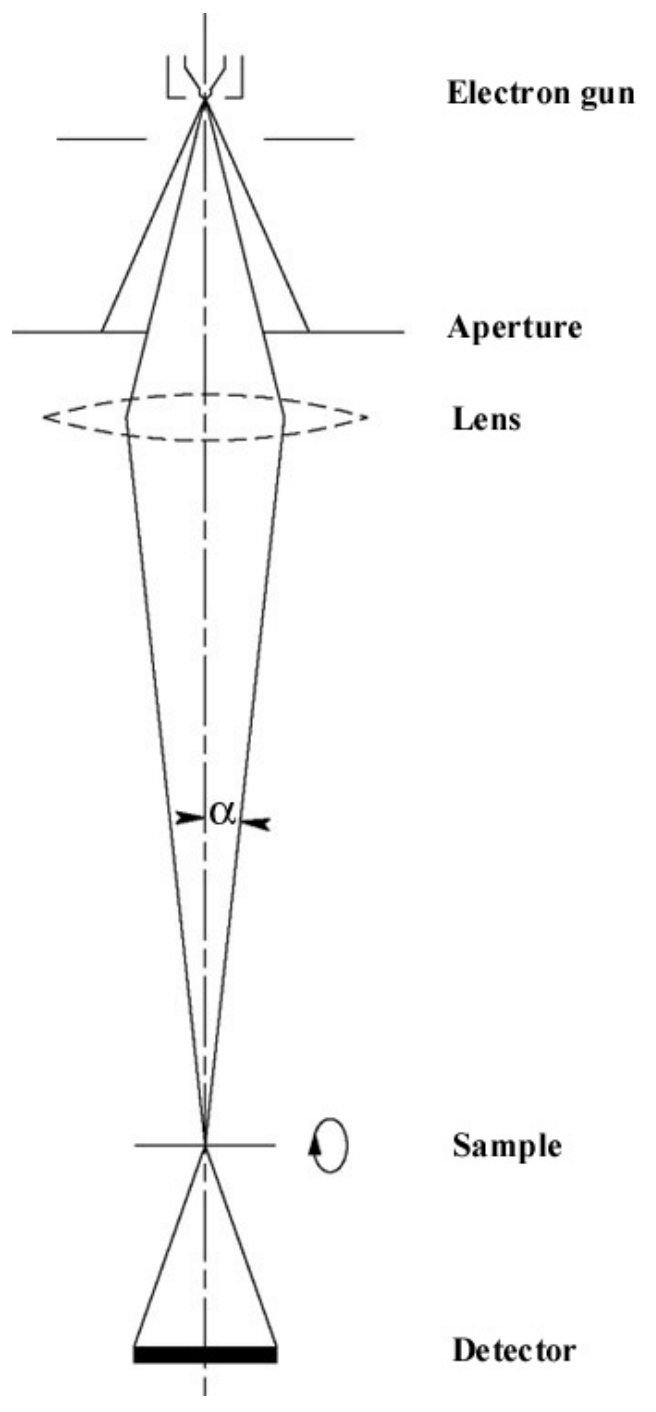

FIG. 1 


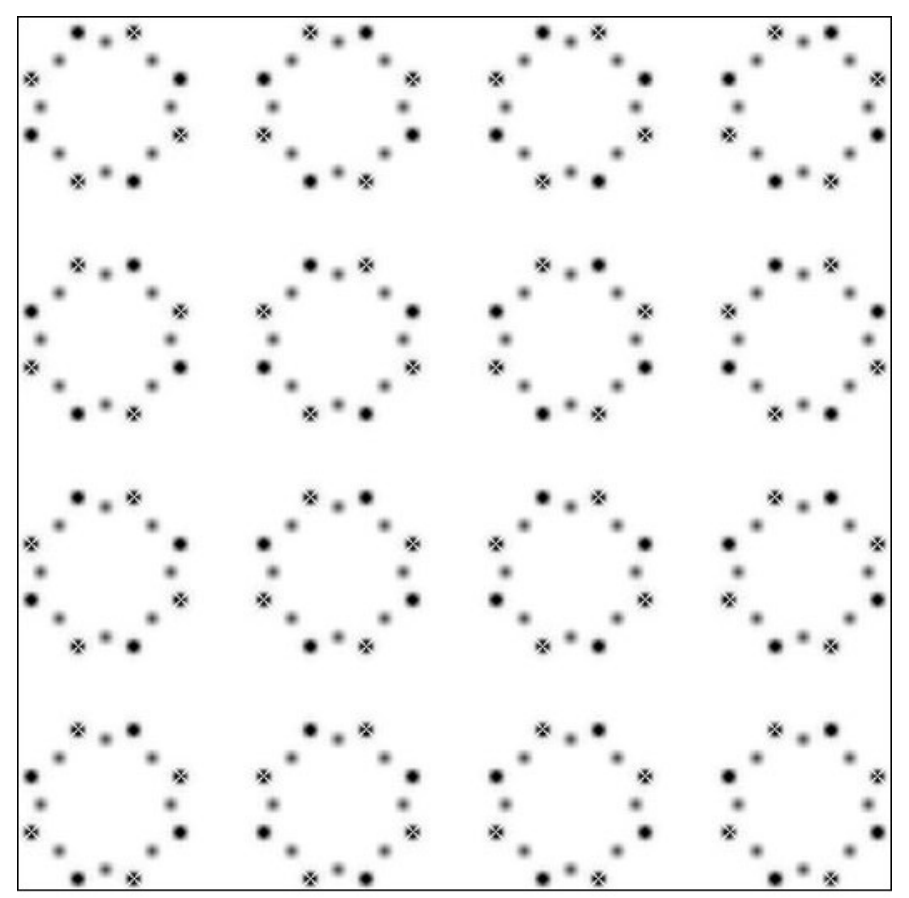

Si * Al * O *

FIG. 2(a)

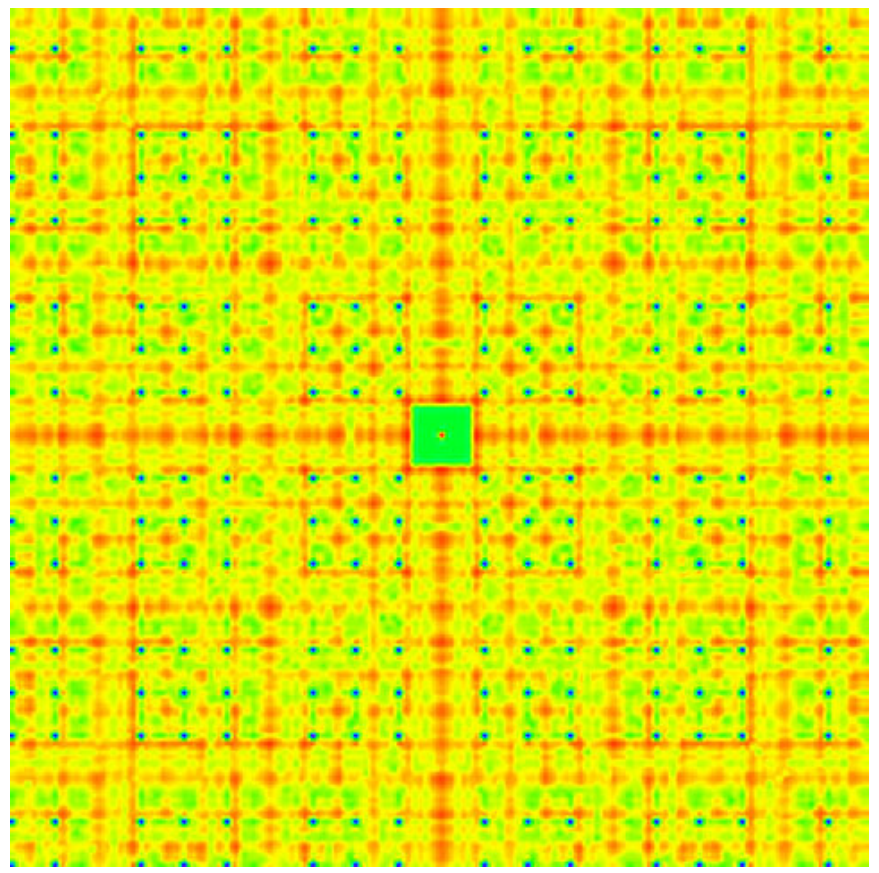

FIG. 2(b) 


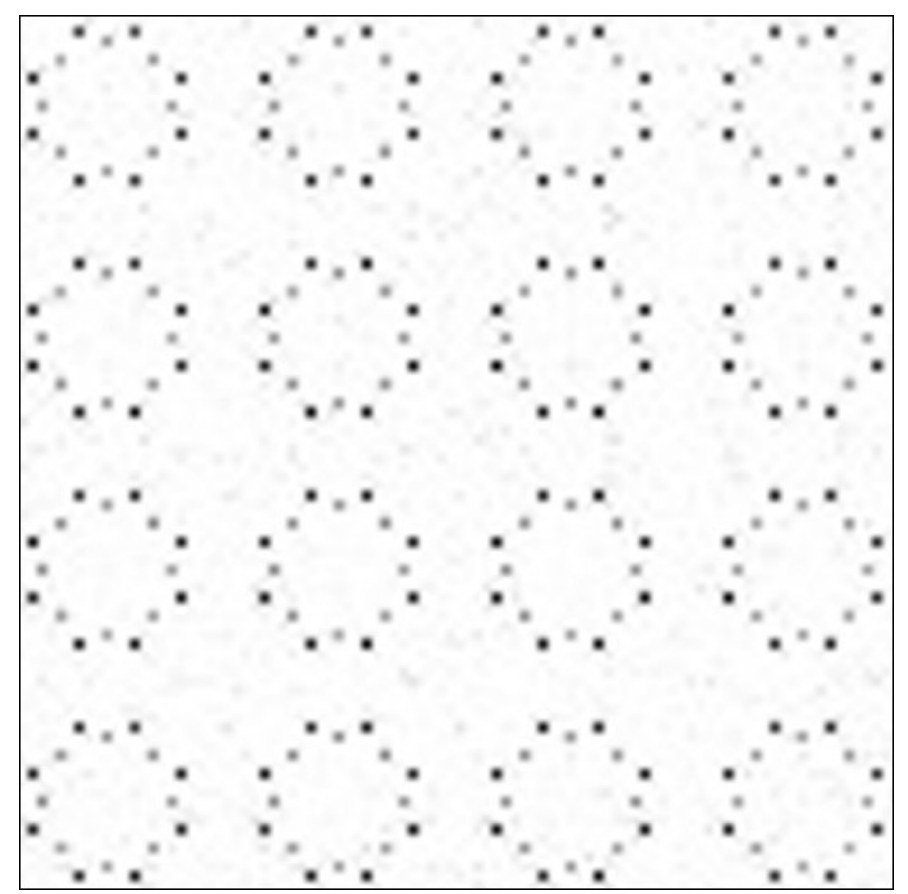

FIG. 2(c)

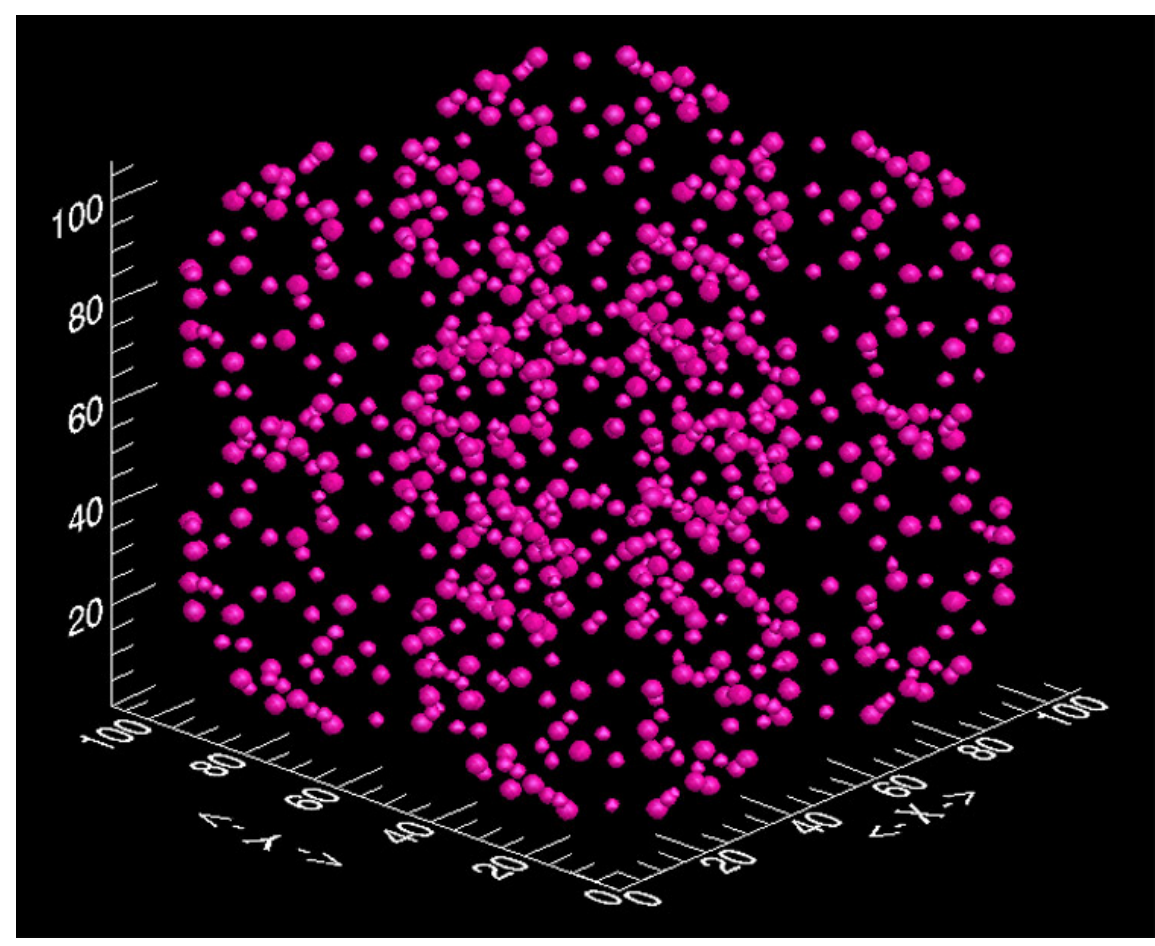

FIG. 2(d) 